Voix et Images

volxetimages

\title{
Anthologies de poésie québécoise
}

\section{François Dumont}

Volume 12, numéro 3 (36), printemps 1987

Yves Beauchemin

URI : https://id.erudit.org/iderudit/200661ar

DOI : https://doi.org/10.7202/200661ar

Aller au sommaire du numéro

Éditeur(s)

Université du Québec à Montréal

ISSN

0318-9201 (imprimé)

1705-933X (numérique)

Découvrir la revue

Citer cet article

Dumont, F. (1987). Anthologies de poésie québécoise. Voix et Images, 12(3),

486-496. https://doi.org/10.7202/200661ar d'utilisation que vous pouvez consulter en ligne.

https://apropos.erudit.org/fr/usagers/politique-dutilisation/ 


\section{Anthologies de poésie québécoise}

\section{par François Dumont, Université Laval}

Pour qui cherche une introduction à la poésie du Québec ou d'ailleurs, l'anthologie semble souvent le type d'ouvrage le plus approprié. Devant l'anthologie, à moins d'une note explicative du compilateur, le lecteur s'attend à un panorama relativement complet et objectif des poètes. L'anthologie est pour l'amateur de poésie une source fondamentale qui indique à partir de quel corpus une lecture d'ensemble personnelle peut être faite. Par ailleurs, l'histoire des anthologies montre ce qu'on a considéré à diverses époques comme valant la peine d'être lu, de même que l'appréciation qu'on a faite des œuvres retenues. L'étude des anthologies québécoises peut donc contribuer à mieux cerner comment la poésie s'est constituée au Québec et quelques-uns des principaux jugements qui ont été portés sur elle.

Il existe de nombreuses anthologies de poésie québécoise. Parmi celles-ci, je ne retiendrai que celles qui s'occupent exclusivement de poésie et qui, au moment de leur parution, prétendaient rendre compte de l'ensemble de la poésie du Québec. J'écarterai donc, par exemple, l'anthologie de la littérature québécoise publiée sous la direction de Gilles Marcotte', l'anthologie de la poésie québécoise du dix-neuvième siècle de John $\mathrm{Hare}^{2}$ et les Stratégies du réel de Nicole Brossard ${ }^{3}$. Je me suis d'autre part limité aux anthologies réalisées au vingtième siècle, excluant par là les florilèges d'Antonin Nantel ${ }^{4}$ ou de Louis-Hippolyte Tachés.

Malgré ces restrictions, restent neuf anthologies de poésie du Québec: celle de Jules Fournier et Olivar Asselin, Anthologie des poètes canadiens $(1920,1933)^{6}$; l'Anthologie de la poésie canadienne d'expression française, canadienne-française puis québécoise de Guy Sylvestre (quatre éditions de

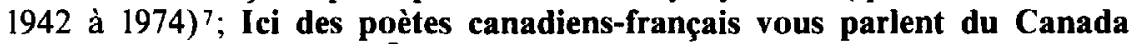
(1944) de Jean Désy ${ }^{8}$; l'Āme de la poésie canadienne-française (1955) de Laure Rièse ${ }^{9}$; la Poésie canadienne (1962) puis du Québec (1968) d'Alain. Bosquet ${ }^{10}$; Feuilles d'érable et fleurs de lys (1965) de Pierre Cabiac ${ }^{11}$; Poètes du Québec (1969) de Jacques Cotnam ${ }^{12}$; trois numéros de la revue Poésie I (Poésie du Québec: les premiers modernes ${ }^{13}$ et la Nouvelle poésie du Québec ${ }^{14}$ parus en 1974, Poètes de l'identité québécoise suivi de les Voix nouvelles ${ }^{15}$ paru en 1982) et enfin la plus récente: la Poésie québécoise (1981) de Laurent Mailhot et Pierre Nepveu ${ }^{16}$.

Ces anthologies ne sont pas d'égale valeur et n'ont pas eu la même diffusion. Quatre d'entre elles se détachent nettement: l'anthologie de Fournier-Asselin, celle de Sylvestre, celle de Bosquet et celle de MailhotNepveu. Pour diverses raisons, les cinq autres méritent moins d'attention. L'anthologie de Jean Désy et celle de Laure Rièse, parues à onze ans d'intervalle, n'ont pas eu un rayonnement comparable à celle de Guy Sylvestre. 
L'anthologie de Pierre Cabiac est un amalgame bâclé et condescendant qui n'a heureusement connu qu'une faible diffusion. Jacques Cotnam, à cause de problèmes de droits d'auteurs, n'a pas réussi de son propre aveu à faire l'anthologie dont il rêvait. D'autre part, son choix est souvent une anthologie de l'anthologie de Sylvestre. Les trois numéros de Poésie I, quoique particulièrement bien faits, ont été soumis aux contraintes éditoriales de la revue et ne prétendent pas à une véritable anthologie. C'est donc à partir des quatre autres anthologies qu'il est possible de suivre la façon dont on a constitué et réévalué le corpus poétique québécois.

Il est étonnant de voir combien l'anthologie de Jules Fournier et Olivar Asselin a mieux vieilli que les manuels scolaires de la même époque. Il faut dire que les auteurs et particulièrement Jules Fournier, concepteur de l'anthologie, n'ont pas voulu faire de leur livre un discours moralisateur, mais bien une sorte de répertoire des poètes les plus intéressants au Canada. Fait assez rare, l'anthologie est conçue - dans la mesure du possible - à partir des propositions des poètes eux-mêmes. Si les auteurs de l'anthologie ont fait un choix de textes qui traduit une certaine échelle de valeurs, ce qui demeure le plus éloquent est, avec l'introduction d'Asselin, la hiérarchie des poètes, qu'on peut établir d'après le nombre de pages accordées à chacun.

De tous les poètes, Émile Nelligan est considéré comme le plus important, tant dans l'édition de 1920 que dans celle de 1933. Le suivent d'assez loin Octave Crémazie, Charles Gill, René Chopin et Louis Fréchette en 1920; Robert Choquette, Crémazie, Fréchette et Alfred DesRochers en 1933. On constate dans le choix des poèmes que Fournier et Asselin se sont largement inspirés des anthologies de Nantel et de Taché comme du Répertoire national. Mais on remarque aussi que la nouvelle poésie intéresse davantage les compilateurs que l'ancienne et qu'à part Nelligan, les poètes du dix-neuvième siècle ne sont pas des "monstres sacrés».

L'introduction rédigée par Olivar Asselin explique bien cet état de choses. Selon Asselin, qui reprend ici l'idée de Jules Fournier (mort avant d'avoir pu terminer l'anthologie) il n'y a pas de littérature canadienne. Jacques Blais ${ }^{17}$ souligne le caractère paradoxal de cette affirmation: ... le texte québécois qui démontre l'inexistence de la littérature québécoise en est lui-même une manifestation ${ }^{18}$. Comme la littérature spécifiquement canadienne est encore à venir, il n'est pas étonnant qu'Olivar Asselin, remaniant l'anthologie en 1933, accorde par exemple plus d'importance à Robert Choquette qu'à Octave Crémazie. Pour Asselin, on ne peut qu'être frappé ... en juxtaposant la "poésie» canadienne de 1800 à 1840 et celle d'aujourd'hui (...) du progrès accompli chez nous sous le double rapport du sentiment et de l'expression poétiques ${ }^{19}$. Malgré cet indéniable progrès, Asselin n'est pas tendre envers l'ensemble de la production poétique québécoise: Pourquoi ne pas reconnaître qu'à tout prendre il serait difficile de trouver, aux époques de civilisation, un nombre égal de rimeurs ayant livré à la publicité, dans un nombre d'années égal, un nombre égal de vers illisibles? ${ }^{20}$ Il est donc clair qu'Asselin, sans doute fidèle au projet initial de Fournier, n'a pas voulu faire l'apologie de la poésie du Québec. Il prend bien soin, au contraire, de répéter plusieurs fois que les 
littérateurs canadiens ne méritent pas qu'on les vénère. Par contre, il tient à montrer qu'il y a un certain nombre de textes dignes d'intérêt et qui pourraient à son avis ouvrir le chemin à une véritable littérature nationale.

Les poètes des années vingt et trente ont tout dè suite vu que le projet d'anthologie de Fournier et Asselin aurait des conséquences importantes sur leur statut de poètes. Asselin souligne l'intérêt de ces auteurs envers son anthologie: Nous leur devons des remerciements publics pour le gracieux empressement qu'ils ont mis à nous fournir les matériaux dont nous avions besoin, et même, en certains cas, ceux dont nous n'avions pas besoin ${ }^{21}$. En même temps qu'il s'insurge contre la suffisance de certains poètes, il insiste pour que puisse se continuer le progrès que l'anthologie permet déjà d'entrevoir. Parallèlement à une ironie mordante, se manifeste donc un grand respect pour les textes, qui pousse Asselin à les reproduire dans leur intégralité. Chaque poète est aussi présenté à son avantage: après une courte biobibliographie figurent souvent quelques extraits d'articles de journaux, toujours élogieux envers l'auteur.

Même si elle ne connut/pas une très large diffusion, cette anthologie en inspira plusieurs autres et en particulier celle de Guy Sylvestre. Celui-ci reprendra en effet bon nombre de poèmes d'abord retenus par Fournier et Asselin. Mais si l'ouvrage de Fournier et Asselin était d'abord une anthologie de poètes, celle de Sylvestre sera fondamentalement une anthologie de poésie.

L'Anthologie de la poésie canadienne d'expression française de Guy Sylvestre paraît en 1942. L'auteur souligne dès le départ l'absence de Paul Morin - celui-ci ayant refusé de céder ses droits d'auteur - dont on découvrira dans les éditions subséquentes qu'il est selon Sylvestre un poète plus important que Nelligan et bon deuxième derrière Alfred DesRochers. Encore une fois, l'introduction donne plusieurs indications quant à l'échelle de valeurs de l'auteur. Guy Sylvestre développe longuement en introduction la thèse d'Henri Bremond selon laquelle il y a une poésie "horizontale", préoccupée par les choses matérielles, et une autre "verticale», essentiellement métaphysique. C'est cette dernière que Sylvestre considère comme la "vraie» poésie et qu'il tente de mettre en valeur dans son anthologie. La conception que Sylvestre se fait de la poésie explique, par exemple, que Rina Lasnier occupe le plus grand nombre de pages (à partir de l'édition de 1963) et que le surréalisme y soit complètement ignoré.

Ce qui est plus difficile à expliquer, c'est la fréquente altération des poèmes: omission de certaines parties (sans indication explicite) qui dénature souvent les textes. Jacques Blais doute qu'il faille attribuer cette pratique à des motifs d'ordre purement esthétique. Il observe en effet des transformations d'ordre idéologique ou carrément politique: ... Sylvestre occulte toute trace des revendications nationales des Québécois: il supprime le sixième et dernier huitain d' $O \hat{O}$ Canada! mon pays! mes amours!" de Georges-Étienne Cartier, où s'exprime un appel aux armes pour libérer le pays du pouvoir d'Albion; il retranche dix des douze quatrains de "Notre langue», de William Chapman, où s'affirme, par défi à l'occupation étrangère, la confiance en la pérennité de 
la langue française au Québec. Par contraste, plusieurs autres pièces se lisent comme des incitations à la bonne entente ou à la conscription ${ }^{22}$.

Cette orientation politique se manifeste aussi clairement dans la nouvelle introduction que Sylvestre publie en 1958. Cette nouvelle introduction, qui sera reprise intégralement jusqu'en 1974, accompagne un changement de titre: il ne s'agit plus de "poésie canadienne d'expression française», mais de poésie "canadienne française». Sylvestre ne franchira le pas vers la «poésie québécoise» qu'en 1974, et encore à reculons. Il ajoutera alors une page à son introduction pour expliquer que "poésie québécoise" est une limitation regrettable, tout comme l'absence de poètes québécois anglophones: ... il s'agit donc, à toutes fins pratiques, d'une anthologie de la poésie québécoise de langue française ${ }^{23}$. On ne saurait mieux nier l'existence du pays québécois, qui est pourtant la principale préoccupation de la plupart des poètes québécois au moment où Sylvestre écrit ces lignes.

Cette distanciation face au discours politique des poètes s'étend aussi au discours québécois sur la poésie. En effet, dans sa nouvelle introduction, Sylvestre se sert bien davantage de points de repère français que de balises proprement québécoises. Il faut aussi signaler que contrairement à Fournier et Asselin, Guy Sylvestre considère que l'âge d'or de la poésie québécoise est passé: Je crains qu'il n'y ait chez la plupart des jeunes poètes canadiens d'aujourd'hui une certaine sécheresse, faut-il dire: une certaine impuissance, qui les empêche de réaliser une oeuvre d'envergure ${ }^{24}$.

Alain Bosquet s'efforcera de prouver le contraire. Celui-ci soutient en effet dans sa propre anthologie, parue en 1962, que tout commence avec Alain Grandbois et culmine dans le présent. Il n'y aurait rien à retenir du début du siècle, et encore moins du dix-neuvième. On pourrait croire que le choix de ne parler que des poètes contemporains ne nie pas pour autant l'intérêt du passé. Mais tel n'est pas le cas. S'il est ...peut-être moins vain de se pencher sur l'ouvre d'Émile Nelligan's, le reste ne vaut carrément pas le déplacement. Ce jugement est d'ailleurs confirmé par l'absence totale du Québec dans l'anthologie de Pierre Seghers (éditeur de l'anthologie de Bosquet): le Livre d'or de la poésie irançaise des origines à $194 \mathbf{4 0}^{25}$. Cetie façon de voir valut à Bosquet de nombreuses critiques. Plusieurs amateurs de poésie québécoise n'apprécièrent guère que soit ainsi jetée aux oubliettes toute l'histoire de la poésie québécoise jusqu'en 1940. Par ailleurs, la majorité d'entre eux semble avoir été flattée par un regard de France si bienveillant à l'égard de la poésie qui s'écrivait alors au Québec. C'est le cas par exemple du poète Gatien Lapointe qui allait jusqu'à dire que la poésie québécoise ... affirmait soudain, et avec véhémence, avec une force jusque-là insoupçonnée, son autonomie ${ }^{27}$. L'autonomie de la littérature québécoise viendrait donc du regard de la France. Cela est d'autant plus paradoxal que Bosquet s'adresse ici d'abord aux Français, bien que son anthologie ait eu une grande audience au Québec. Un peu comme dans Maria Chapdelaine, on parle des Québécois mais pas à eux. Le Québec est l'objet, sans être ni destinateur, ni destinataire. Pour Bosquet, cette mise à distance serait garante d'une plus grande universalité: L'universalité, vue par des yeux canadiens, a quelque chance d'être 
une universalité canadienne; ce risque est atténué en France: il y a bien longtemps que notre universalité à nous ne se confond pas avec les aspirations ou les drames de notre pays ${ }^{28}$...

Alain Bosquet réévalue à son tour la production poétique québécoise. I1 donne le premier la parole à Claude Gauvreau et établit une nouvelle hiérarchie, qui fait d'abord se succéder Alphonse Piché, Saint-Denys Garneau, Rina Lasnier, Sylvain Garneau, Alain Grandbois et Gatien Lapointe. Ce dernier, bien qu'il soit représenté comme l'un des plus importants, est pourtant décrit comme ... un poète de grand avenir ${ }^{29}$. Ce qui nous ramène en quelque sorte au jugement porté quarante ans plus tôt par Jules Fournier et Olivar Asselin: le meilleur de la poésie québécoise reste à venir.

Sans doute en partie pour réagir au point de vue de Bosquet, Laurent Mailhot et Pierre Nepveu publient en 1981 la Poésie québécoise. La poésie du Québec au dix-neuvième siècle n'aurait pas d'intérêt? Tout ne commencerait vraiment qu'en 1940? Qu'à cela ne tienne! Les auteurs remontent jusqu'à Marc Lescarbot, c'est-à-dire presque deux siècles plus tôt que Fournier et Asselin, qui considéraient pourtant avoir inclus des vers ...comme probablement jamais autre anthologiste ne fut ni ne sera dans la triste obligation d'en publier ${ }^{30}$. Mailhot et Nepveu ne reprennent pas pour autant le choix de Sylvestre, choix auquel ils réagiront aussi en dévaluant presque systématiquement les "vedettes» établies par leur prédécesseur.

Guidés par le désir de faire un «monument» à la gloire de la poésie québécoise, Mailhot et Nepveu s'inspireront surtout de Fournier et Asselin dans leur choix de poètes. Pour ce qui est du choix des poèmes eux-mêmes, ... Mailhot-Nepveu ont substitué la rhétorique mystique à la rhétorique patriotique (...) La magie remplace la religion et le fantastique, la politique ${ }^{31}$. Il s'agit donc en bonne partie d'une réécriture de l'histoire de la poésie québécoise en fonction du présent et plus encore du passé récent. En effet, le temps fort de la poésie québécoise (établi d'après le nombre de pages) résiderait dans les années cinquante et soixante, en particulier à l'Hexagone (qui co-édite d'ailleurs l'anthologie). Pour ce qui est de l'espace accordé, on peut même observer une nette courbe ascendante jusqu'à l'Hexagone (assortie de commentaires expliquant que tel ou tel poète "annonce l'Hexagone»), qui se met à décroître avec Paul Chamberland.

Richard Giguère ${ }^{32}$ a souligné à quel point ce "monument" était essentiellement composé de poètes montréalais. Les Écrits des Forges de TroisRivières, par exemple, ne sont même pas mentionnés en introduction. La poésie de Québec est aussi très peu considérée. Mais ce sont surtout les femmes qui sont les grandes oubliées de cette anthologie. On le comprend pour le dix-neuvième siècle, mais cela s'étend aussi à la poésie contemporaine. Par exemple, des trente-six poètes les plus importants, trois femmes seulement: Anne Hébert, Rina Lasnier et Nicole Brossard (de justesse). Sur les cent soixante et onze poètes que réunit l'anthologie, vingt-six femmes. On remarque l'absence de Thérèse Renaud, qui publia pourtant le premier recueil d'inspiration surréaliste au Québec, ou le fait que même pour les années soixante-dix, on ait retenu trois fois plus d'hommes que de femmes. 
Malgré cela, l'anthologie de Mailhot et Nepveu constitue certainement la meilleure anthologie de poésie québécoise actuellement disponible. Moins datée que celle de Fournier et Asselin, moins "personnalisée" que celle de Sylvestre, moins limitée que celle de Bosquet, elle donne la parole à des voix variées qu'elle met bien en valeur. Il s'agit à la limite d'une sorte de répertoire, qui prétend jusqu'à un certain point à l'objectivité, tout en s'identifiant assez nettement à l'institution littéraire québécoise (entendue dans son sens montréalais...). .

Si le temps fort est celui de l'Hexagone, les poètes les plus importants sont ceux qui ont ajouté un "écart esthétique» à l'«horizon d'attente» des années soixante: Paul-Marie Lapointe et Gaston Miron, bons premiers; Jacques Brault et Paul Chamberland, qui suivent de près Émile Nelligan, Saint-Denys Garneau et Anne Hébert. À propos de cette dernière, on peut remarquer le prestige des romanciers. En effet, non seulement Anne Hébert est-elle considérée comme la poète la plus importante, mais encore Gérard Bessette, Jacques Godbout, Marie-Claire Blais et même Roch Carrier figurent tous dans l'anthologie ${ }^{33}$. A propos de Nelligan, on peut le voir passer, d'une anthologie à l'autre, de triste victime de la névrose ${ }^{34}$ à extraordinaire adolescent ${ }^{35}$, pour finalement devenir, plus qu'un malade ou un mythe, un écrivain, pour qui le poème est un texte à travailler ${ }^{36}$. Quant à Saint-Denys Garneau, notons qu'il devance ici Alain Grandbois, ce que Jacques Blais explique par la conception prosaïque de la poésie qui se dégage de l'anthologie:

D'après les notices de présentation et les textes retenus, la définition du poème idéal actuel selon Mailhot-Nepveu se lirait un peu comme suit. Toute rhétorique exclue, indifférent aux modes et aux écritures d'école, le poème idéal a, pour tonalité dominante, l'angoisse; pour énergie, la violence; pour régulateur, l'ironie. Son intention: dire l'essentiel d'une existence individuelle. Ses moyens: un langage rompu, dénudé, rigoureux, incisif, qui règle le délire, purifie et pétrifie les phantasmes. On y a accès d'emblée. En somme: le classicisme, en pleine modernité. Et comme le poème à la neutralité forcée se révèle prosaïque, on aboutit à une équation orwelienne: la poésie, c'est la prose ${ }^{37}$.

A partir de l'exemple des ouvrages de Fournier-Asselin, Sylvestre, Bosquet et Mailhot-Nepveu, on peut constater que le discours des anthologies recoupe sur plusieurs points celui des manuels scolaires de littérature. Clément Moisan ${ }^{38}$ a proposé une problématique générale d'analyse de ces manuels, problématique dont je retiendrai quatre propositions.

La première est que le manuel est un discours idéologique. Le cas de Guy Sylvestre montre bien qu'il en est de même pour l'anthologie. Nous avons vu comment, chez Sylvestre, le titre, l'introduction, le choix des auteurs et des textes tout comme la "manipulation" des poèmes avaient de claires implications politiques. Le discours idéologique n'est pas aussi évident dans chaque anthologie, mais il est toujours présent, ne serait-ce que sous la forme de présupposés fondant la discrimination des auteurs et des textes. 
La deuxième proposition est que le manuel est un discours axiologique sur la littérature. L'anthologie ne comprend pas, comme le manuel, d'« explications de textes" qui orientent la lecture, mais elle est le lieu par excellence de ce que Pierre Kuentz ${ }^{39}$ a appelé la "canonisation" des auteurs, des textes et des mouvements littéraires. A la manière du discours idéologique, le discours axiologique sur la littérature n'est souvent qu'implicite, dans le type de textes retenus et dans la hiérarchie des auteurs. Il est aussi parfois explicité en introduction, comme chez Fournier-Asselin et chez Guy Sylvestre.

La troisième proposition de Clément Moisan est que le manuel est une réponse apportée à d'autres manuels qui le précèdent ou qui sont en concurrence avec lui. Les quatre cas que j'ai retenus montrent bien que les anthologies de poésie québécoise forment une sorte de système clos: Fournier et Asselin répondent à Nantel; Sylvestre répond à Fournier et Asselin; Bosquet à Sylvestre; Mailhot et Nepveu répondent à la fois à Bosquet et à Sylvestre, tout en faisant un clin d'œil à Fournier et Asselin.

Quatrième proposition: les manuels font partie du processus de constitution et de légitimation de la littérature. Pour ce qui est des anthologies québécoises, il serait plus précis de parler de "littérature nationale». L'intention avouée d'Olivar Asselin était en effet de permettre l'élaboration d'une future littérature nationale. Guy Sylvestre écrivait quant à lui dans son introduction: On a souvent discuté de l'existence d'une littérature canadienne; cette existence ne peut plus être mise en doute, comme le prouverait, si c'était nécessaire, cette anthologie 40 . Alain Bosquet disait lui aussi vouloir fournir quelques preuves (...) de l'originalité de la poésie canadienne ${ }^{41}$. Mailhot et Nepveu évoquent le but de sauvegarder un patrimoine national ${ }^{42}$, même s'ils ajoutent que cela n'a pas été leur principale préoccupation. Dans les anthologies de poésie québécoise, on insiste donc moins sur la légitimité de la littérature que sur la légitimité de la littérature nationale et, en dernière analyse, sur la légitimité de la nation elle-même. C'est ce qui expliquerait que les anthologies québécoises privilégient toujours le point de vue historique: il semble qu'il faille prouver, aux autres comme à nous-mêmes, que nous avons vraiment une histoire, et donc que nous sommes une nation.

Dans le cadre de sa quatrième proposition, Clément Moisan insiste sur le fait que le processus de légitimation amène, de façon sous-jacente, la reconnaissance du pouvoir lui-même de consacrer, c'est-à-dire la reconnaissance de la légitimité de l'institution littéraire. Ceci s'applique spécifiquement aux anthologies québécoises, dont les auteurs prétendent, comme dans les manuels, parler au nom de l'institution littéraire. Il existe cependant dans d'autres pays, en France notamment, de nombreuses anthologies "d'auteur», qui n'ont pas d'équivalent au Québec pour le moment. Citons entre autres la célèbre anthologie de Paul Éluard: le Meilleur choix de poèmes est celui que l'on fait pour soi ${ }^{43}$ ou, parue ces dernières années: Une anthologie de la poésie française de Jean-François Revel ${ }^{44}$, d'où sont exclus par exemple Aragon, Lautréamont et Queneau. Éluard et Revel n'ont pas hésité à proposer un choix de poèmes très personnel, en marge des canons de l'institution littéraire française. 
ẼTUDES 493

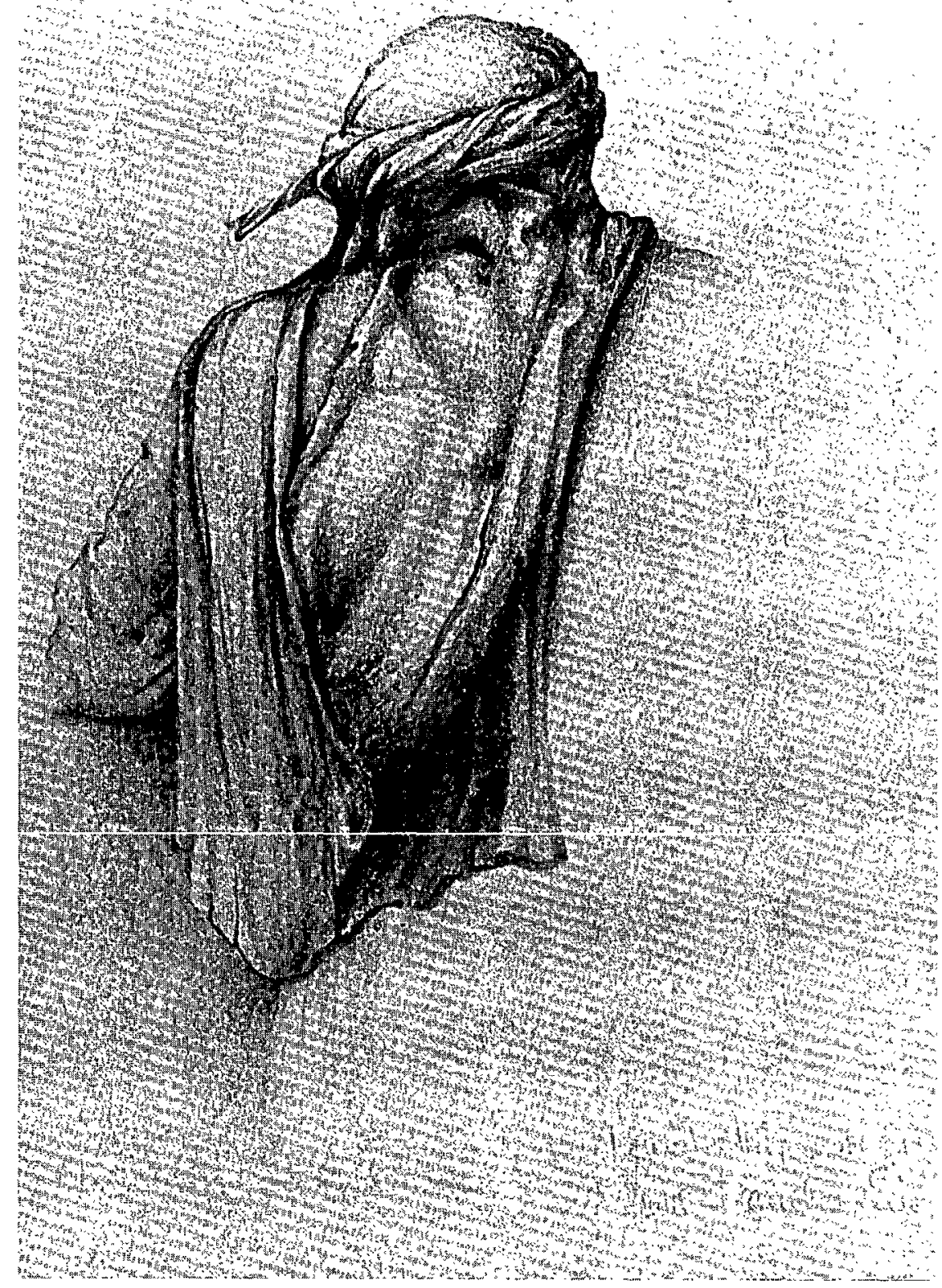


Bien qu'à plusieurs égards les anthologies tiennent un discours semblable à celui des manuels, elles s'en écartent par ailleurs sur certains points. Ainsi, comme nous venons de le voir à propos des ouvrages d'Éluard et Revel, le lien entre les anthologies et l'institution littéraire n'est pas toujours aussi net que dans le cas des manuels. Quelque part entre le manuel et l'essai, l'anthologie permet à son auteur d'avancer du nouveau, plutôt que de se limiter à reproduire le discours d'autres instances, comme le font les auteurs de manuels. Ce fut le cas de Guy Sylvestre, qui fit redécouvrir l'œuvre de Jean-Aubert Loranger. En effet, s'il faut en croire Gilles Marcotte, on l'aurait peut-être oublié définitivement si dans son Anthologie de la poésie canadienne-française, Guy Sylvestre ne lui avait redonné la parole ${ }^{45}$. Semblablement, Alain Bosquet retiendra des textes de Claude Gauvreau, bien avant qu'un certain consensus ne l'y incite. De façon générale, le type de discours de l'anthologie diffère du type de discours du manuel du fait que le premier n'est pas exclusivement didactique.

L' «instance anthologique" a beaucoup plus de pouvoir sur la poésie que sur tout autre genre littéraire. Il s'est fait bien plus d'anthologies de poésie que d'anthologies d'extraits de romans, essais ou pièces de théâtre. C'est qu'on a l'habitude de considérer qu'un recueil de poésie n'est pas une œuvre, mais un ensemble d'œuvres détachables et autonomes. Pour ce qui est des autres genres, quand on cite un extrait, on situe habituellement le contexte. En poésie, on va jusqu'à faire comme si le poème lui-même était décomposable sans perdre sa nature. Nous avons vu comment Sylvestre se permettrait de transformer des poèmes sans même l'indiquer. Mailhot et Nepveu "corrigeront" eux aussi des poèmes, mais en indiquant toujours l'endroit où les coupures ont été effectuées. Cette pratique du découpage fait souvent en sorte que les versions corrigées deviennent les plus diffusées. Le pouvoir de l'anthologiste prime dans ces cas sur le pouvoir de l'auteur quant à la teneur même de ses propres textes.

L'anthologie est donc un médiateur de première importance pour la poésie. C'est d'abord l'anthologie qui consacre les poètes; qui distingue les poètes importants des poètes secondaires: qui dégage les mouvements poétiques les plus significatifs, le véritable sens de l'histoire de la poésie et, au bout du compte, ce qu'est, comme ce que doit être la poésie. Tout cela n'étant souvent pas exprimé en tant que discours subjectif, prend une force de persuasion d'autant plus considérable. Mais l'anthologie n'est pas pour autant uniquemènt un instrument de pouvoir. Elle est certes aussi un moyen privilégié pour sortir la poésie (et ici la poésie québécoise en particulier) de son "circuit restreint».

Août 1986

1. Gilles Marcotte, et coll., Anthologie de la littérature québécoise, Montréal, La Presse, III (1895-1837), 1979, 498 p., IV (1937-1952), 1980, 463 p.

2. John Hare, Anthologie de la poésie québécoise du XIXe siècle, Montréal, Hurtubise HMH, "Cahiers du Québec", 1979, 410 p.

3. Nicole Brossard, Les Stratégies du réel/The Story so far 6, Montréal, La Nouvelle Barre du jour et Toronto, The Coach House Press, 1979, 341 p. 
4. Antonin Nantel, Les Fleurs de la poésie canadienne, Montréal, Beauchemin, 1869, $134 \mathrm{p}$; $1896,225 \mathrm{p}$.

5. Louis-Hyppolyte Taché, La Poésie franco-canadienne, Saint-Hyacinthe, Imprimerie du Courrier de Saint-Hyacinthe, 1881, $286 \mathrm{p}$.

6. Jules Fournier et Olivar Asselin, Anthologie des poètes canadiens, Montréal, (s.é.), 1920, 309p.; Granger Frères Limitée, éditeurs, 1933, 229 p.

7. Guy Sylvestre, Anthologie de la poésie canadienne d'expression française, Montréal, Éditions Bernard Valiquette, 1942, 141 p.; Anthologie de la poésie canadiennefrançaise, Montréal, Éditions Beauchemin, 1958, XIII, 298 p.; 1963, 376 p.; 1974, $412 \mathrm{p}$.

8. Jean Désy, Ici des poètes canadiens nous parlent du Canada, (s. I.), Americ éditeur, (1944), 191 p.

9. Laure Rièse, L’Âme de la poésie canadienne-française, Toronto, MacMillan, 1955, XXXI, 263 p.

10. Alain Bosquet, La Poésie canadienne, Montréal et Paris, Éditions Seghers et HMH, (1962), 222 p.; Poésie du Québec, Paris et Montréal, Seghers HMH, (1971), 271 p.

11. Pierre Cabiac, Feuilles d'érable et fleurs de lys, Paris, Éditions de la Diaspora française, 1965, t. I, 249 p.; t II, 1966, 244 p.

12. Jacques Cotman, Poètes du Québec, Montréal, Fides, 1968, 222 p.

13. Jacques Rancourt, Poésie du Québec: les premiers modernes, Poésie I, no 35, janvierfévrier $1974,119 \mathrm{p}$.

14. Jacques Rancourt, la Nouvelle poésie du Québec, Poésie I, no 36, mars-avril 1974, $120 \mathrm{p}$.

15. Jacques Rancourt, Poètes de l'identité québécoise suivi de les Voix nouvelles, Poésie I. no 96-98, mars-avril 1982, $224 \mathrm{p}$.

16. Laurent Mailhot et Pierre Nepveu, la Poésie québécoise, Montréal, Les Presses de l'Université du Québec, les Éditions de l'Hexagone, 1981, 714 p.

17. Jacques Blais s'est intéressé de façon soutenue aux anthologies de poésie québécoise. C'est lui qui a rédigé tous les articles du Dictionnaire des œeuvres littéraires du Québec qui concernent des anthologies de poésie. Il a également publié une analyse de l'anthologie de Mailhot et Nepveu dans Lettres québécoises, comme nous le verrons plus loin. Signalons que Jacques Blais est lui-même l'auteur d'une anthologie; Anthologie de la poésie québécoise de 1900 à 1950, Université Laval, département des littératures, Première partie, 1900-1934, août 1984, 80 p.; Deuxième partie, 1935-1950, octobre 1984,51 p. Le compilateur a choisi des poèmes tenant chacun dans une seule page et reproduits intégralement. La particularité de ce choix est de se limiter à un seul poème pari recucil, depuisis Emile Nelligan at son aure jusqu'à État mixte de Claude Gauvreau. La chronologie est basée ici sur les publications plutôt que sur la date de naissance des poètes. Apparaissent aussi occasionnellement des inédits et des extraits de prose lyrique.

18. Jacques Blais, "Anthologie des poètes canadiens", Dictionnaire des quvres littéraires du Québec, Tome II, p. 47.

19. Jules Fournier et Olivar Asselin, op. cit, 1933, p. 10-11.

20. Ibid., p. 11.

21. Ibid., p. 16.

22. Jacques Blais, "Anthologie de la poésie canadienne d'expression française", DOLQ, Tome III, p. 55.

23. Guy Sylvestre, Anthologie de la poésie québécoise, op. cit., p.X.

24. Ibid., p. XXIII.

25. Alain Bosquet, Poésie du Québec, op. cit., p. 14.

26. Pierre Seghers, le Livre d'or de la poésie française des origines à 1940, Verviers, Marabout université, 1972, 464 p. Paradoxalement, dans la récente anthologie de 
Ahmed Ben Othman et Jean-Pierre Darmon: Cent poèmes pour la liberté, Paris, Le Cherche midi éditeur, 1984, 190 p., ce sont Louis Fréchette et William Chapman qui représentent l'ensemble de la poésie québécoise...

27. Gatien Lapointe, "Anthologie de la poésie canadienne", Livres et auteurs canadiens, 1966, p. 88.

28. Alain Bosquet, op. cit., p. 12.

29. Ibid., p. 200.

30. Jules Fournier et Olivar Asselin, op. cit., p. 16.

31. Jacques Blais, «la Poésie québécoise des origines à nos jours", Lettres québécoises, no 23, automne 1981 , p. 43.

32. Richard Giguère, compte rendu paru dans Estuaire, no 23, printemps 1982, p. 113-116.

33. Le prestige du roman se double du prestige des prix littéraires, sur lesquels Mailhot et Nepveu insistent beaucoup. Déjà, dans la dernière édition de l'anthologie de Sylvestre, on pouvait voir Marie-Claire Blais devenir une poète de première importance à la suite de l'obtention du prix Médicis pour Une saison dans la vie d'Emmanuel.

34. Jules Fournier et Olivar Asselin, op. cit., p. 240.

35. Guy Sylvestre, op. cit., p. XV.

36. Laurent Mailhot et Pierre Nepveu, op. cit., p. 160.

37. Jacques Blais, op. cit., p. 44.

38. Dans un ouvrage à paraître: Melançon, Joseph, Clément Moisan et Max Roy, les Processus de formation littéraire dans l'enseignement classique au Québec, Québec, Éditions du CRELIQ (Centre de recherche en littérature québécoise), Université Laval, Coll. "Travaux de recherche".

39. Pierre Kuentz, "L'envers du texte», Littérature, no 7, octobre 1972, p. 3-26.

40. Guy Sylvestre, op. cit., p. XI.

41. Alain Bosquet, op. cit., p. 13.

42. Laurent Mailhot et Pierre Nepveu, op. cit., p. 9.

43. Paul Éluard, Le Meilleur choix de poèmes est celui que lon fait pour soi, Paris, Editions du Sagittaire, 1947, $383 \mathrm{p}$.

44. Jean-François Revel, Une anthologie de la poésie française, Paris, Laffont, coll. “Bouquins", 1984, $660 \mathrm{p}$.

45. Jean-Aubert Loranger, Les Atmosphères suivi de Poëmes, préface de Gilles Marcotte, Montréal, HMH, coll. «Sur parole», 1970, p. 9. 